\title{
A New Prognostic Tool for Patients Undergoing Radiotherapy plus Upfront Transurethral Resection for Bladder Cancer
}

\author{
LISA MANIG $^{1}$, STEFAN JANSSEN ${ }^{1}$, STEVEN E. SCHILD ${ }^{2}$ and DIRK RADES ${ }^{1}$ \\ ${ }^{1}$ Department of Radiation Oncology, University of Lübeck, Lübeck, Germany; \\ ${ }^{2}$ Department of Radiation Oncology, Mayo Clinic, Scottsdale, AZ, U.S.A.
}

\begin{abstract}
Aim: To create a tool to estimate overall survival $(O S)$ of patients with non-metastatic bladder cancer. Patients and Methods: Eight parameters were retrospectively evaluated for $O S$ in 42 patients undergoing radiotherapy and upfront transurethral resection for bladder cancer. The prognostic tool included those parameters that were significant on both univariate and multivariate analyses. The tool was based on the 3-year OS rate divided by 10. Patient scores were calculated by adding the points for each significant parameter. Results: On univariate analysis, 3year OS was associated with gender $(p=0.017)$, pack years $(p=0.009)$ and Karnofsky performance score (KPS) $(p<0.001)$. On Cox regression, pack years $(p=0.007)$ and KPS $(p<0.001)$ remained significant and were incorporated into the tool. Three prognostic groups were designated with 6, 10-11 and 15 points with 3-year OS-rates of $0 \%, 34 \%$ and $83 \%$, respectively $(p<0.001)$. Conclusion: A tool was developed to estimate the OS of patients with bladder cancer to improve individualization of treatment.
\end{abstract}

Bladder-sparing treatment including radiotherapy plus transurethral resection has become more popular recently, particularly for elderly patients and those with significant comorbidities $(1,2)$. Radical cystectomy, which is still considered the standard approach in many institutions, is associated with a considerable risk of significant complications including perioperative death (3-5).

Although multimodality treatment including transurethral resection of tumour followed by radiotherapy ideally

This article is freely accessible online.

Correspondence to: Professor Dirk Rades, Department of Radiation Oncology, University of Lübeck, Ratzeburger Allee 160, D-23552 Lübeck, Germany. Tel: +49 45150045401, e-mail: rades.dirk@ gmx.net

Key Words: Bladder cancer, radiotherapy, transurethral resection, overall survival, prognostic tool. supplemented with simultaneous chemotherapy is less invasive than radical cystectomy, it is still a challenging treatment regimen and may not be tolerated by many patients with bladder cancer (1). To avoid over- or undertreatment of patients, the treatment regimen should be optimally tailored to each patient's situation. Such an individualized approach should consider several aspects, including the patient's survival prognosis. However, estimation of the remaining lifespan is often difficult for the treating physician and would be facilitated with a simple prognostic tool that can be easily used during clinical routine and provides the physician the ability to predict a patient's survival time.

This study was conducted to create just such a predictive tool, created for patients with bladder cancer who cannot or do not wish to undergo radical cystectomy and prefer a bladder-preserving approach including radiotherapy and upfront transurethral resection of the bladder tumour.

\section{Patients and Methods}

Eight clinical parameters were retrospectively evaluated for potential correlation with overall survival (OS) in 42 patients undergoing transurethral resection of the bladder plus subsequent radiotherapy for primary treatment of non-metastatic bladder cancer (Table I). All patients had received an equivalent dose in 2 Gy-fractions of $>55 \mathrm{~Gy}$. The radiation regimen was $59.4 \mathrm{~Gy}$ in 33 fractions of $1.8 \mathrm{~Gy}$ in 37 patients, $57.8 \mathrm{~Gy}$ in 32 fractions of 1.8 Gy in two patients, 56 Gy in 28 fractions of 2.0 Gy in one, 60 Gy in 30 fractions of $2.0 \mathrm{~Gy}$ in one and $63 \mathrm{~Gy}$ in 35 fractions of 1.8 Gy in one, respectively. Thirty-two patients (76\%) received chemotherapy during the radiotherapy course. The chemotherapy included cisplatin alone in 15 , paclitaxel alone in 11 , cisplatin/paclitaxel in three, carboplatin alone in two and vinflunine alone in one.

Those clinical parameter that were found to be significantly associated $(p<0.05)$ with OS on both univariate (Kaplan-Meier estimates plus log-rank test) and multivariate analyses (Cox regression model) were included in the prognostic tool. The tool was designed to predict an individual patient's OS-probability at 3 years following radiotherapy. It was based on the 3-year OS-rates of those clinical parameters which were significantly associated with OS on univariate and multivariate analyses of this study, 
Table I. Distribution of clinical parameters investigated in this study.

\begin{tabular}{lcc}
\hline & $\begin{array}{c}\text { Number of } \\
\text { patients }\end{array}$ & $\begin{array}{c}\text { Proportion } \\
(\%)\end{array}$ \\
\hline Gender & & \\
$\quad$ Female & 18 & 43 \\
$\quad$ Male & 24 & 57 \\
Age at radiotherapy & & \\
$\quad \leq 77$ Years & 26 & 62 \\
$\quad>77$ Years & 16 & 38 \\
T-Stage & & \\
T1-2 & 18 & 43 \\
T>2a & 15 & 36 \\
T3-4 & 9 & 21 \\
N-Stage & & \\
N0 & 37 & 88 \\
N1-2 & 5 & 12 \\
Grading & & \\
G2 & 7 & 17 \\
G3 & 35 & 83 \\
Pack years & & \\
$\quad<40$ & 35 & 83 \\
$\quad \geq 40$ & 7 & 17 \\
Simultaneous chemotherapy & & \\
$\quad$ No & 10 & 24 \\
Yes & 32 & 76 \\
Karnofsky performance score & & \\
$\quad \leq 70$ & 34 & \\
$>70$ & & \\
\hline
\end{tabular}

divided by 10 . The scores for individual patients were calculated by adding the points obtained for each of the significant parameters

\section{Results}

On univariate analysis, better 3-year OS was significantly associated with female gender $(p=0.017),<40$ pack years $(p=0.009)$ and a Karnofsky performance score (KPS) of $>70(p<0.001)$ (Table II). In the Cox regression model, the number of pack years [risk ratio $(\mathrm{RR})=6.85$; 95\% confidence interval $(\mathrm{CI})=1.72-28.36 ; p=0.007]$ and KPS $(\mathrm{RR}=12.88 ; 95 \%-\mathrm{CI}=3.22-58.82 ; \quad p<0.001) \quad$ remained significant, whereas gender $(\mathrm{RR}=1.54 ; 95 \%-\mathrm{CI}=0.43-6.31$; $p=0.52$ ) did not.

The prognostic tool included pack years and KPS, the parameters that were significant in both univariate and multivariate analyses. Considering the 3-year OS-rates, the points ascribed were 7 for $<40$ pack years, 3 for $\geq 40$ pack years, 8 for KPS $>70$ and 3 for KPS $\leq 70$, respectively. Thus, total scores ranged between 6 and 15. Three prognostic groups were designated, with $6,10-11$ and 15 points, with 3year OS rates of $0 \%, 34 \%$ and $83 \%$, respectively, and median OS duration of 2, 36 and 76 months, respectively $(p<0.001$, Figure 1).
Table II. Univariate analyses of overall survival (OS) including the 3year OS rate.

\begin{tabular}{|c|c|c|}
\hline & $\begin{array}{c}\text { OS at } \\
3 \text { years }(\%)\end{array}$ & $p$-Value \\
\hline \multicolumn{3}{|l|}{ Gender } \\
\hline Female & 76 & \\
\hline Male & 60 & 0.017 \\
\hline \multicolumn{3}{|c|}{ Age at radiotherapy } \\
\hline$\leq 77$ Years & 58 & \\
\hline$>77$ Years & 79 & 0.10 \\
\hline \multicolumn{3}{|l|}{ T-Stage } \\
\hline $\mathrm{T} 1-2$ & 61 & \\
\hline $\mathrm{T}>2 \mathrm{a}$ & 72 & \\
\hline T3-4 & 74 & 0.98 \\
\hline \multicolumn{3}{|l|}{$\mathrm{N}$-Stage } \\
\hline No & 64 & \\
\hline $\mathrm{N} 1-2$ & 100 & 0.58 \\
\hline \multicolumn{3}{|l|}{ Grading } \\
\hline $\mathrm{G} 2$ & 54 & \\
\hline G3 & 70 & 0.95 \\
\hline \multicolumn{3}{|l|}{ Pack years } \\
\hline$<40$ & 74 & \\
\hline$\geq 40$ & 29 & 0.009 \\
\hline \multicolumn{3}{|c|}{ Simultaneous chemotherapy } \\
\hline No & 55 & \\
\hline Yes & 71 & 0.52 \\
\hline \multicolumn{3}{|c|}{ Karnofsky performance score } \\
\hline$\leq 70$ & 30 & \\
\hline$>70$ & 75 & $<0.001$ \\
\hline
\end{tabular}

Significant $p$-values are shown in bold.

\section{Discussion}

Considerable efforts have been made to improve the prognoses of patients with bladder cancer (6-10). Many of these patients are not able to withstand radical cystectomy, which is associated with severe complications in almost one-third of patients (3-5). The alternative approach including transurethral resection of the tumour and local radiotherapy with or without radio-sensitising chemotherapy is also aggressive and may result in serious adverse events, mainly of the bladder and the rectum. When selecting a bladder-preserving treatment approach, one should always consider the patient's particular situation, including their survival prognosis. Patients with a short remaining lifespan may not benefit from an aggressive approach with a high radiation dose and concurrent chemotherapy; a shorter radiation program without chemotherapy may be more appropriate. On the other hand, for patients with a more favourable survival prognosis, local and locoregional control of the disease is more important. In these patients, a more aggressive approach including higher radiation doses and chemotherapy appears justified in order to improve the outcome. 


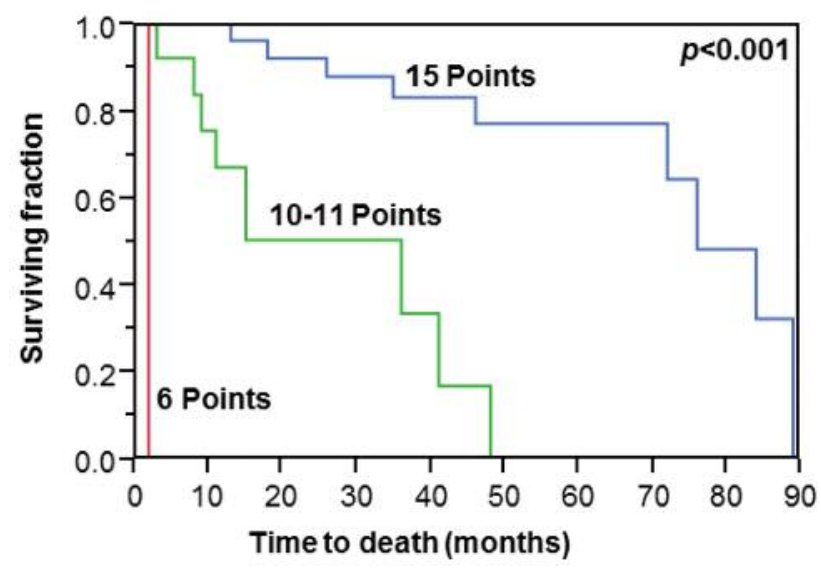

Figure 1. Comparison of the three groups defined using the prognostic tool based on pack years and Karnofsky performance score (KaplanMeier curves). The p-value (univariate analysis) was obtained from the log-rank test.

These considerations make it clear that it is important to know a patient's survival prognosis in order to choose the best treatment regimen for each patient. A prognostic tool designed specifically to estimate the survival of this particular patient group is needed. Such tools have been developed for patients with bladder cancer in other situations, such as locally recurrent and metastatic disease $(11,12)$. However, a tool for definitive bladder-preserving treatment has been lacking. To close this gap, the present study was performed.

In this study, a prognostic tool for estimation of survival of patients undergoing organ-preserving treatment for bladder cancer was created. It included those clinical parameters that proved to be significant on both univariate and multivariate analyses, namely pack years and KPS. Based on these two parameters, three prognostic groups were designed with significantly different 3-year OS rates and median OS times. For the 6-point group, the 3-year OS rate was $0 \%$, and the median OS duration only 2 months. These patients should be treated with a very short course of radiotherapy without concurrent chemotherapy and may even be considered for best supportive care alone. Patients of the 10-11 point group had a relatively poor 3 -year OS rate of $34 \%$. Their treatment regimen should be chosen on an individual basis considering the patient's preference weighing improved local control versus an increased risk of serious adverse events. The radiotherapy dose may be lower than 59.4 Gy in 33 fractions and concurrent chemotherapy may be omitted, depending on the patient's choice. For the 15 -point group, the 3 -year OS rate was $83 \%$, and the median OS duration 76 months. These patients would likely benefit from a more aggressive approach including a radiation dose of 59.4 Gy in 33 fractions supplemented with concurrent chemotherapy, since these patients live long enough to be at risk of developing a local or locoregional recurrence, which cannot be effectively treated with a second course of radiotherapy or radio-chemotherapy.

When following these recommendations, physicians should be aware that the predictive tool was generated from retrospective data and bears the risk of including hidden selection biases. However, since patients with bladder cancer undergoing organ-preserving treatment are uncommon, prospective trials with a sufficient statistical power cannot be expected in the near future. Moreover, the findings of the present study that a lower number of pack years and a better KPS are significantly associated with longer OS agree with data regarding other oncological situations reported in the literature and, therefore, show consistency $(11,13-15)$. The fact that a lower number of pack years results in better treatment outcomes was demonstrated for other tumours including head-and-neck cancer $(13,14)$. A positive association with greater KPS and improved OS was previously found in patients with locally recurrent or metastatic bladder cancer $(11,15)$.

In conclusion, a prognostic tool was developed to be able to estimate the survival of patients with bladder cancer undergoing a bladder-preserving treatment approach including radiotherapy with upfront transurethral resection of the tumour. This new prognostic tool can improve the individualization of the treatment for this particular group of patients with bladder cancer.

\section{Conflicts of Interest}

On behalf of all Authors, the corresponding Author states that there is no conflict of interest related to this study.

\section{References}

1 Rodel C, Grabenbauer GG, Kühn R, Papadopoulos T, Dunst J, Meyer M, Schrott KM and Sauer R: Combined-modality treatment and selective organ preservation in invasive bladder cancer: long-term results. J Clin Oncol 20: 3061-3071, 2002.

2 Maebayashi T, Ishibashi N, Aizawa T, Sakaguchi M, Sato K, Matsui T, Yamaguchi K and Takahashi S: Radiotherapy for muscle-invasive bladder cancer in very elderly patients. Anticancer Res 36: 4763-4769, 2016.

3 Konety BR, Allareddy V and Herr H: Complications after radical cystectomy: analysis of population-based data. Urology 68: 5864, 2006.

4 Manoharan M, Ayyathurai R and Soloway MS: Radical cystectomy for urothelial carcinoma of the bladder: an analysis of perioperative and survival outcome. BJU Int 104: 1227-1232, 2009.

5 Koga F and Kihara K: Selective bladder preservation with curative intent for muscle-invasive bladder cancer: a contemporary review. Int J Urol 19: 388-401, 2012. 
6 Lea MA, Guzman Y and Desbordes C: Inhibition of growth by combined treatment with inhibitors of lactate dehydrogenase and either phenformin or inhibitors of 6-phosphofructo-2-kinase/ fructose-2,6-bisphosphatase 3. Anticancer Res 36: 1479-1488, 2016.

7 Hancock BM, McGuire KL, Tsuji S, Reil K, Hernandez V, Giacalone MJ and Godbey WT: A single intravesical instillation of VAX014 inhibits orthotopic superficial bladder tumor implantation to increase survival. Anticancer Res 36: 6243-6248, 2016.

8 Nakagawa YU, Nagaya H, Miyata T, Wada Y, Oyama T and Gotoh A: Piperazine-based alpha-1 AR blocker, naftopidil, selectively suppresses malignant human bladder cells via induction of apoptosis. Anticancer Res 36: 1563-1570, 2016.

9 Kilari D, Iczkowski KA, Pandya C, Robin AJ, Messing EM, Guancial E and Kim ES: Copper transporter-CTR1 expression and pathological outcomes in platinum-treated muscle-invasive bladder cancer patients. Anticancer Res 36: 495-501, 2016.

10 Sakaguchi M, Maebayashi T, Aizawa T, Ishibashi N and Saito $\mathrm{T}$ : Clinical results for bladder cancer treated by radiotherapy without concurrent standard chemotherapy. Anticancer Res 36: 5519-5525, 2016.

11 Manig L, Käsmann L, Janssen S and Rades D: Predicting survival after irradiation of metastases from transitional carcinoma of the bladder. Anticancer Res 36: 6663-6665, 2016.
12 Manig L, Janssen S, Schild SE and Rades D: A new prognostic instrument specifically designed for patients irradiated for recurrent carcinoma of the bladder. In Vivo 31: 435-438, 2017.

13 Gillison ML, Zhang Q, Jordan R, Xiao W, Westra WH, Trotti A, Spencer S, Harris J, Chung CH and Ang KK: Tobacco smoking and increased risk of death and progression for patients with p16-positive and p16-negative oropharyngeal cancer. J Clin Oncol 30: 2102-2111, 2012.

14 Granata R, Miceli R, Orlandi E, Perrone F, Cortelazzi B, Franceschini M, Locati LD, Bossi P, Bergamini C, Mirabile A, Mariani L, Olmi P, Scaramellini G, Potepan P, Quattrone P, Ang KK and Licitra L: Tumor stage, human papillomavirus and smoking status affect survival of patients with oropharyngeal cancer: an Italian validation study. Ann Oncol 23: 1832-1837, 2012.

15 Rades D, Manig L, Janssen S and Schild SE: Concurrent chemotherapy improves the overall survival of patients irradiated for locally recurrent bladder cancer. Anticancer Res 37: 14851488, 2017. 